\title{
High Intraoperative Pulse Pressure Is A Risk Factor For A Postoperative Organ Injury In A Cohort of Abdominal Surgery Patients: An Exploratory Study
}

Zbigniew Putowski ( $\sim$ putowski.zbigniew@gmail.com )

Medical University of Silesia: Slaski Uniwersytet Medyczny w Katowicach

\section{Szymon Czajka}

Medical University of Silesia: Slaski Uniwersytet Medyczny w Katowicach

Łukasz J. Krzych

Medical University of Silesia: Slaski Uniwersytet Medyczny w Katowicach

\section{Research}

Keywords: hemodynamic monitoring, general surgery, pulse pressure, postoperative complications

Posted Date: August 4th, 2021

DOl: https://doi.org/10.21203/rs.3.rs-770176/v1

License: (c) (i) This work is licensed under a Creative Commons Attribution 4.0 International License.

Read Full License 


\section{Abstract}

Background: Both intraoperative hypotension and hypertension have been reported to increase the occurrence of either acute kidney injury (AKI), myocardial infraction (MI) or stroke. However, intraoperative pulse pressure's (PP) impact on the latter complications remains relatively unknown.

Methods: This is a cohort study in which patients who underwent abdominal surgery between 1 October 2018 and 15 July 2019 in university hospital in Katowice were included in the analysis. Pre- and intraoperative data, including blood pressure measurements, were acquired via medical charts. Several PP thresholds were applied: $>50,>55,>60,>65,>70,>75,>80,>85,>90 \mathrm{mmHg}$. Additionally, by analysing the maximal PP during procedures, a cut-off point for the occurrence of outcomes was estimated. Postoperative complications were defined as occurrence of either AKI, MI or stroke. Univariable and multivariable analyses were performed to assess PP's relationship with hypoperfusive organ injury.

Results: 508 patients were included in the analysis. Hypoperfusion was present in 38 (7.5\%) cases. ROC curve analysis estimated a cut-off point of $>74 \mathrm{mmHg}$ of maximal PP to be associated with the outcomes. PP values above $65 \mathrm{mmHg}$ onward were included in the multivariable statistical models. A model in which $\mathrm{PP}>90 \mathrm{mmHg}(\mathrm{OR}=4.21 ; 95 \% \mathrm{Cl} 1.73-10.24 ; \mathrm{p}=0.0015)$ was included, had the best predicting value in predicting hypoperfusive injury. Apart of PP, intraoperative hypotension, presence of chronic arterial hypertension and procedure duration were independently associated with postoperative complications.

Conclusions: High intraoperative pulse pressure may be associated with the occurrence of hypoperfusionrelated organ injury. However, the effect of high pulse pressure should be confirmed in other non-cardiac populations to prove generalizability of our results.

\section{Background}

Hypoperfusion-related organ injury is a fairly frequent perioperative complication [1-4]. Intraoperative hypotension $(\mathrm{IOH})$ has been linked with postoperative myocardial injury (MI), acute kidney injury (AKI) and stroke [1-3]. Perioperative Quality Initiative (POQI) consensus statement on intraoperative blood pressure underlines that mean arterial pressure (MAP) below $60-70 \mathrm{mmHg}$ and systolic blood pressure (SBP) below $100 \mathrm{mmHg}$ are associated with hypoperfusion-related organ injury and death [4]. However, hypertensive events during surgery may also worsen outcome, as intraoperative episodes of SBP above $160 \mathrm{mmHg}$ have been correlated with the risk of myocardial injury and infraction [4]. Lastly, diastolic blood pressure (DBP) below $50 \mathrm{mmHg}$ is also reported to be harmful [5].

Although ambulatory pulse pressure (PP) is considered as one of the best predictors of cardiovascular risk [6] it has been poorly investigated in the perioperative period. The association between high preoperative PP values and the relationship with postoperative complications (mainly myocardial infraction, acute kidney injury and stroke) has been explored mostly in cardiosurgical patient populations. POQI has called for further research on the matter in non-cardiac surgery [7]. Therefore, in an exploratory 
fashion, we sought to verify whether elevated intraoperative PP values are associated with hypoperfusion-related organ injury in abdominal surgery.

\section{Methods}

The data used in this study comes from a prospective cohort study published previously by our team [8]. We screened 576 consecutive patients who underwent abdominal surgery between 1 October 2018 and 15 July 2019 in a university hospital. Procedures of organ procurement $(n=11)$, reoperations $(n=24)$, procedures performed in local anaesthesia or monitored anaesthetic supervision $(n=33)$, and those classified as immediate according to the NCEPOD Classification of Intervention [9] $(n=14)$ were excluded (Fig. 1). Demographic and medical data were recorded, including sex, age, weigh, height, comorbidities and its pharmacological treatment, according to the ICD 10 criteria. Body mass index (BMI) and Charlson Comorbidity Index (CCl) were subsequently calculated. Type and duration of anaesthesia, and type, duration and urgency of surgery were recorded. Perioperative risk was assessed based on individual patient's risk, according to the American Society of Anaesthesiology (ASA) physical status (PS) classification [10], and procedural risk, according to the European Society of Cardiology and European Society of Anaesthesiology recommendations [11]. Primary arterial hypertension was diagnosed based on medical records.

Figure 1. Flow diagram for the patient selection process

Systolic (SBP) and diastolic blood pressure (DBP) were measured on a non-dominant arm with an automated oscillometric non-invasive BP monitoring device (Dräger Infinity Gamma XL) with a cuff of appropriate size depending on patient's arm circumference and recorded in five-minute intervals during anaesthesia, from the first pre-induction measurement until the last measurement during recovery from anaesthesia in the operating theatre. Mean (MAP) blood pressure values were automatically calculated. Pulse pressure was calculated as the difference between SBP and DBP. A need for norepinephrine (NE) use, its doses and duration of infusion, together with intraoperative fluid balance were analysed.

As PP revolves usually around values of $40 \mathrm{mmHg}$ and that, based on other studies on clinical consequences of abnormal PP, we distinguished the following absolute PP thresholds: $>50 \mathrm{mmHg},>55$ $\mathrm{mmHg},>60 \mathrm{mmHg},>65 \mathrm{mmHg},>70 \mathrm{mmHg},>75 \mathrm{mmHg},>80 \mathrm{mmHg},>85 \mathrm{mmHg}$ and $>90 \mathrm{mmHg}[5$, 12-14]. Additionally, by analysing the maximal PP during a procedure, the best cut-off point associated with the occurrence of outcomes was estimated. Moreover, we analysed the occurrence of high systolic (defined as SBP > $160 \mathrm{mmHg}$ [15]), low diastolic (defined as DBP $<50 \mathrm{mmHg}$ [16]) and low mean arterial pressure (defined as MAP $<60 \mathrm{mmHg}$ [17]. We excluded pre-induction measurements in order to assess only those blood pressure values that occurred during anaesthesia.

In the postoperative period, the incidents of hypoperfusion of vital organs were recorded, and included the occurrence of AKI, stroke and MI according to their international definitions [18-20]. This composite endpoint was considered as the outcome. 
STROBE (STrengthening the Reporting of OBservational studies in Epidemiology) statement was applied for appropriate reporting [21].

Statistical analysis was performed using MedCalc Statistical Software version 18.1 (MedCalc Software Ltd., Ostend, Belgium). Continuous variables were expressed as median and interquartile range (IQR). Qualitative variables were expressed as absolute values and/or percent. Between-group differences for quantitative variables were assessed using Mann-Whitney U-test. Their distribution was verified with Shapiro-Wilk test. Chi-square test were applied for qualitative variables. The correlation was assessed using Spearman's rank correlation coefficient. ROC curve analysis was implemented to assess the relationship between composite outcome and maximal PP values and pre-induction PP values. In order to control for potential confounding factors, we used multivariable logistic regression with all variables that achieved p-value of less than 0.1 in univariable analysis. If applicable, odds ratios (OR) and Area Under the Receiver Operating Characteristics (AUROC) with their $95 \%$ confidence intervals $(\mathrm{Cl})$ were calculated. All tests were two-tailed. A ' $p$ ' value $<0.05$ was considered statistically significant.

\section{Results}

Total number of patients included in the analysis was 508, 239 (46\%) were male. The median age of participants was 65 years (IQR 46-68). The majority (90.4\%) of subjects underwent elective surgery. Older age, higher ASA-PS class, higher $\mathrm{CCl}$ were found to be significant preoperative risk factors for occurrence of hypoperfusion outcome. Detailed preoperative population characteristics are presented on Table 1, whereas intraoperative population characteristics are presented on Table 2. The composite primary outcome was diagnosed in 38 (7.5\%) patients, including 32 cases of AKI (6.3\%), 3 cases of MI $(0.6 \%)$ and one event of stroke (0.2\%). Pre-induction PP was not associated with the outcome (Table 1$)$. 
Table 1

Preoperative population characteristics

\begin{tabular}{|c|c|c|c|c|}
\hline Variable & All $n=508(100.0)$ & $\begin{array}{l}\text { Outcome }(-) \\
n=470\end{array}$ & $\begin{array}{l}\text { Outcome (+) } \\
n=38\end{array}$ & P-value \\
\hline Age (years) & $62(46-68)$ & $61(45-68)$ & $67(62-75)$ & $\begin{array}{l}p< \\
0.01\end{array}$ \\
\hline Male (n) & $239(46.0)$ & $219(46.6)$ & $20(52.6)$ & 0.4 \\
\hline Height (cm) & $169(162-176)$ & $169(161-176)$ & $168(164-171)$ & 0.2 \\
\hline Weight (kg) & $73(63-84)$ & $73(63-84)$ & $73(58-86)$ & 0.9 \\
\hline $\mathrm{BMI}\left(\mathrm{kg} \mathrm{m}^{-2}\right)$ & $25.7(22.5-29.2)$ & $25.6(22.5-29.0)$ & $27.1(21.9-29.8)$ & 0.3 \\
\hline Arterial hypertension & $234(46.1)$ & $205(43.6)$ & $29(76.3)$ & $\begin{array}{l}p< \\
0.01\end{array}$ \\
\hline $\begin{array}{l}\text { Pre-induction SBP } \\
(\mathrm{mmHg})\end{array}$ & $140(125-155)$ & $140(125-153)$ & $142.5(130-155)$ & 0.1 \\
\hline $\begin{array}{l}\text { Pre-induction MAP } \\
(\mathrm{mmHg})\end{array}$ & $\begin{array}{l}101.7(92.3- \\
110.0)\end{array}$ & $\begin{array}{l}101.7(92.0- \\
110.0)\end{array}$ & $\begin{array}{l}101.5(95.0- \\
110.0)\end{array}$ & 0.6 \\
\hline Pre-induction PP (mmHg) & $57.0(49.0-67.0)$ & $56.5(48.5-65.0)$ & $60.0(50.0-75.0)$ & 0.3 \\
\hline ASA-PS I/II & $293(57.7)$ & $281(59.8)$ & $12(31.6)$ & $\begin{array}{l}p< \\
0.01\end{array}$ \\
\hline ASA-PS III/IV/V & $215(42.3)$ & $189(40.2)$ & $26(68.4)$ & $\begin{array}{l}\mathrm{p}< \\
0.01\end{array}$ \\
\hline $\mathrm{CCl}$ (pts) & $3(1-5)$ & $3(1-5)$ & $5(3-7)$ & $\begin{array}{l}p< \\
0.01\end{array}$ \\
\hline Premedication & $305(60.0)$ & $284(60.4)$ & $21(55.3)$ & 0.5 \\
\hline Elective surgery & $459(90.4)$ & $427(90.9)$ & $32(84.2)$ & 0.1 \\
\hline
\end{tabular}


Table 2

Intraoperative population characteristics

\begin{tabular}{|c|c|c|c|c|}
\hline Variable & $\begin{array}{l}\text { All } n=508 \\
(100.0)\end{array}$ & $\begin{array}{l}\text { Outcome }(-) \\
n=470\end{array}$ & $\begin{array}{l}\text { Outcome } \\
(+) \\
n=38\end{array}$ & $\begin{array}{l}\mathrm{P} \text { - } \\
\text { value }\end{array}$ \\
\hline General + regional anesthesia $(n)$ & $40(7.8)$ & $31(6.6)$ & $9(23.7)$ & $\begin{array}{l}p< \\
0.01\end{array}$ \\
\hline Invasive blood pressure monitoring (n) & $83(16.3)$ & $67(14.7)$ & $14(36.8)$ & $\begin{array}{l}p< \\
0.01\end{array}$ \\
\hline Procedure risk I (n)* & $45(8.9)$ & $44(9.4)$ & $1(2.6)$ & 0.1 \\
\hline Procedure risk II $(n)^{\star}$ & $335(65.9)$ & $314(66.8)$ & $21(55.3)$ & 0.1 \\
\hline Procedure risk III (n)* & $128(25.2)$ & $112(23.8)$ & $16(42.1)$ & 0.01 \\
\hline Oncological procedure $(\mathrm{n})$ & $245(48.2)$ & $219(46.6)$ & $26(68.4)$ & $\begin{array}{l}p< \\
0.01\end{array}$ \\
\hline Catecholamine use (n) & $227(44.7)$ & $197(41.9)$ & $30(78.9)$ & $\begin{array}{l}p< \\
0.01\end{array}$ \\
\hline $\begin{array}{l}\text { Time of catecholamine administration from } \\
\text { the induction of anesthesia (min) }\end{array}$ & $\begin{array}{l}40.0(20.0- \\
75.0)\end{array}$ & $\begin{array}{l}40.0(20.0- \\
80.0)\end{array}$ & $\begin{array}{l}37.5(15.0- \\
60.0)\end{array}$ & 0.4 \\
\hline Catecholamine dose $\left(\mu \mathrm{g} \mathrm{kg}^{-1} \mathrm{~min}^{-1}\right)$ & $\begin{array}{l}0.063 \\
(0.042- \\
0.090)\end{array}$ & $\begin{array}{l}0.054 \\
(0.042- \\
0.090)\end{array}$ & $\begin{array}{l}0.070 \\
(0.048- \\
0.091)\end{array}$ & 0.3 \\
\hline Procedure duration (min) & $\begin{array}{l}230.0 \\
(130.0- \\
340.0)\end{array}$ & $\begin{array}{l}215.0 \\
(120.0- \\
330.0)\end{array}$ & $\begin{array}{l}372.5 \\
(235.0- \\
492.0)\end{array}$ & ¿. 001 \\
\hline Fluid dose $\left(\mathrm{mL} \mathrm{kg}^{-1} \mathrm{~h}^{-1}\right)$ & $\begin{array}{l}6.78(5.1- \\
8.76)\end{array}$ & $\begin{array}{l}6.78(5.16- \\
8.76)\end{array}$ & $\begin{array}{l}6.674 .74- \\
8.58)\end{array}$ & 0.6 \\
\hline Mean arterial pressure $(\mathrm{mmHg})$ & $\begin{array}{l}83.33 \\
(78.33- \\
88.33)\end{array}$ & $\begin{array}{l}83.33 \\
(78.33- \\
88.33)\end{array}$ & $\begin{array}{l}85.17 \\
(78.33- \\
90.00)\end{array}$ & 0.4 \\
\hline $\begin{array}{l}\text { Pre-induction pulse pressure } \\
(\mathrm{mmHg})\end{array}$ & $\begin{array}{l}57.0(49.0- \\
67.0)\end{array}$ & $\begin{array}{l}56.5(48.5- \\
65.0)\end{array}$ & $\begin{array}{l}60.0(50.0- \\
75.0)\end{array}$ & 0.2 \\
\hline $\begin{array}{l}\text { Minimal pulse pressure during anesthesia } \\
(\mathrm{mmHg})\end{array}$ & $\begin{array}{l}30.0(25.0- \\
35.0)\end{array}$ & $\begin{array}{l}30.0(25.0- \\
35.0)\end{array}$ & $\begin{array}{l}25.0(24.0- \\
33.0)\end{array}$ & 0.1 \\
\hline
\end{tabular}

MAP: Mean Arterial Pressure; SBP: Systolic Blood Pressure; DBP: Diastolic Blood Pressure * according to European Society of Cardiology and European Society of Anaesthesiology recommendations [10]. 


\begin{tabular}{|c|c|c|c|c|}
\hline Variable & $\begin{array}{l}\text { All } n=508 \\
(100.0)\end{array}$ & $\begin{array}{l}\text { Outcome (-) } \\
n=470\end{array}$ & $\begin{array}{l}\text { Outcome } \\
(+) \\
n=38\end{array}$ & $\begin{array}{l}\mathrm{P}- \\
\text { value }\end{array}$ \\
\hline $\begin{array}{l}\text { Median pulse pressure during anesthesia } \\
(\mathrm{mmHg})\end{array}$ & $\begin{array}{l}45.3 \\
(40.00- \\
53.00)\end{array}$ & $\begin{array}{l}45.0(40.0- \\
51.8)\end{array}$ & $\begin{array}{l}51.8(46.5- \\
60.0)\end{array}$ & $\begin{array}{l}p< \\
0.01\end{array}$ \\
\hline $\begin{array}{l}\text { Maximal pulse pressure during anesthesia } \\
(\mathrm{mmHg})\end{array}$ & $\begin{array}{l}65.0(58.0- \\
77.0)\end{array}$ & $\begin{array}{l}65.0(56.0- \\
75.0)\end{array}$ & $\begin{array}{l}82.5(70.0- \\
92.0)\end{array}$ & $\begin{array}{l}p< \\
0.01\end{array}$ \\
\hline MAP $<60 \mathrm{mmHg}$ during anesthesia $(\mathrm{n})$ & $124(24.4)$ & $107(22.8)$ & $17(44.7)$ & $\begin{array}{l}p< \\
0.01\end{array}$ \\
\hline $\mathrm{SBP}>160 \mathrm{mmHg}$ during anesthesia $(\mathrm{n})$ & $111(28.0)$ & $98(20.9)$ & $13(34.2)$ & $\begin{array}{l}P= \\
0.05\end{array}$ \\
\hline $\mathrm{DBP}<50 \mathrm{mmHg}$ during anesthesia $(\mathrm{n})$ & $151(29.7)$ & $133(28.3)$ & $18(47.4)$ & $\begin{array}{l}P= \\
0.01\end{array}$ \\
\hline \multicolumn{5}{|c|}{ MAP: Mean Arterial Pressure; SBP: Systolic Blood Pressure; DBP: Diastolic Blood Pressure } \\
\hline
\end{tabular}

In patients who developed hypoperfusion-related organ injury, PP negatively correlated with DBP than patients without postoperative complications (Table 3).

Table 3

Correlation between pulse pressure and systolic blood pressure, diastolic blood pressure and mean arterial pressure

\begin{tabular}{|lll|}
\hline Variable & $\begin{array}{l}\text { Pulse pressure; } \\
\text { Outcome }(-)(n=470)\end{array}$ & $\begin{array}{l}\text { Pulse pressure; } \\
\text { Outcome }(+)(n=38)\end{array}$ \\
\hline Systolic blood pressure & $R=0.635 ; p<0.01$ & $R=0.594 ; p<0.01$ \\
\hline Diastolic blood pressure & $R=-0.239 ; p<0.01$ & $R=-0.578 ; p<0.01$ \\
\hline Mean arterial pressure & $R=0.082 ; p=0.07$ & $R=-0.315 ; p=0.05$ \\
\hline Pre-induction pulse pressure & $R=0.472 ; p<0.01$ & $R=0.513 ; p<0.01$ \\
\hline The values are Spearman's rank correlation coefficients and ' $p$ ' values \\
\hline
\end{tabular}

Maximal PP registered over the course of procedure was associated with the outcome (AUROC $=0.75 ; p<$ 0.001), with a cut-off point of $>74 \mathrm{mmHg}$ (Fig. 2).

Figure 2. ROC curve analysis of maximal PP values registered over the course of procedure.

In univariable analyses, all PP thresholds, except from $>50 \mathrm{mmHg}$, were statistically significant predictors of hypoperfusion (Fig. 3). In multivariable logistic regressions, $\mathrm{PP}>50 \mathrm{mmHg},>55 \mathrm{mmHg}$ and $>60$ 
$\mathrm{mmHg}$ were not included in the final statistical models. It was discovered that PP above $90 \mathrm{mmHg}$ predicted hypoperfusion-related organ injury with the highest accuracy, even after adjustment for intraoperative hypertension (Table 4). Low DBP ( $<50 \mathrm{mmHg}$ ) and high SBP ( $>160 \mathrm{mmHg}$ ) were not significant in the multivariable models. 
Table 4

Multivariate logistic regression models in predicting the occurrence of hypoperfusive outcome

\begin{tabular}{|c|c|c|c|c|c|c|c|c|}
\hline $\begin{array}{l}\text { Variable* } \\
\downarrow\end{array}$ & $\underset{\rightarrow}{\text { Model }}$ & $\begin{array}{l}\mathrm{PP}>65 \\
\mathrm{mmHg} \\
(1 / 0)\end{array}$ & $\begin{array}{l}\mathrm{PP}>70 \\
\mathrm{mmHg} \\
(1 / 0)\end{array}$ & $\begin{array}{l}\mathrm{PP}>74 \\
\mathrm{mmHg} \\
(1 / 0)\end{array}$ & $\begin{array}{l}\mathrm{PP}>75 \\
\mathrm{mmHg} \\
(1 / 0)\end{array}$ & $\begin{array}{l}\mathrm{PP}>80 \\
\mathrm{mmHg} \\
(1 / 0)\end{array}$ & $\begin{array}{l}\mathrm{PP}>85 \\
\mathrm{mmHg} \\
(1 / 0)\end{array}$ & $\begin{array}{l}\mathrm{PP}>90 \\
\mathrm{mmHg} \\
(1 / 0)\end{array}$ \\
\hline \multirow{3}{*}{\multicolumn{2}{|c|}{ Pulse pressure }} & 3.14 & 2.53 & 2.43 & 2.13 & 2.68 & 3.08 & 4.21 \\
\hline & & $\begin{array}{l}(1.23- \\
8.04)\end{array}$ & $\begin{array}{l}(1.16- \\
5.53)\end{array}$ & $\begin{array}{l}(1.13- \\
5.27)\end{array}$ & $\begin{array}{l}(1.00- \\
4.52) ;\end{array}$ & $\begin{array}{l}(1.24- \\
5.81)\end{array}$ & $\begin{array}{l}\text { (1.37- } \\
6.89)\end{array}$ & $\begin{array}{l}(1.73- \\
\text { 10.24); }\end{array}$ \\
\hline & & $\begin{array}{l}p= \\
0.0168\end{array}$ & $p=0.02$ & $\begin{array}{l}p= \\
0.0238\end{array}$ & $\begin{array}{l}p= \\
0.0489\end{array}$ & $\begin{array}{l}P= \\
0.0124\end{array}$ & $\begin{array}{l}p= \\
0.0063\end{array}$ & $\begin{array}{l}P= \\
0.0015\end{array}$ \\
\hline \multirow{3}{*}{\multicolumn{2}{|c|}{$\begin{array}{l}\text { Chronic arterial } \\
\text { hypertension }(1 / 0)\end{array}$}} & 3.17 & 3.22 & 3.19 & 3.22 & 3.02 & 3.08 & 3.41 \\
\hline & & $\begin{array}{l}(1.37- \\
7.36) ; \mathrm{p}\end{array}$ & $\begin{array}{l}(1.39- \\
7.44)\end{array}$ & $\begin{array}{l}(1.38- \\
7.39)\end{array}$ & $\begin{array}{l}(1.38- \\
7.50)\end{array}$ & $\begin{array}{l}(1.29- \\
7.06)\end{array}$ & $\begin{array}{l}(1.33- \\
7.17)\end{array}$ & $\begin{array}{l}(1.48- \\
7.88)\end{array}$ \\
\hline & & & $\begin{array}{l}p= \\
0.0062\end{array}$ & $\begin{array}{l}p= \\
0.0068\end{array}$ & $\begin{array}{l}p= \\
0.0066\end{array}$ & $\begin{array}{l}P= \\
0.0109\end{array}$ & $\begin{array}{l}P= \\
0.0089\end{array}$ & $\begin{array}{l}P= \\
0.004\end{array}$ \\
\hline \multirow{3}{*}{\multicolumn{2}{|c|}{$\begin{array}{l}\text { Procedure duration } \\
\text { (per } 1 \text { minute) }\end{array}$}} & 1.006 & 1.006 & 1.006 & 1.0066 & 1.006 & 1.006 & 1.006 \\
\hline & & $\begin{array}{l}(1.003- \\
1.008)\end{array}$ & $\begin{array}{l}\text { (1.003- } \\
1.008)\end{array}$ & $\begin{array}{l}(1.003- \\
1.008)\end{array}$ & 1.008); & $\begin{array}{l}(1.003- \\
1.008)\end{array}$ & $\begin{array}{l}(1.003- \\
1.008)\end{array}$ & $\begin{array}{l}(1.003- \\
1.008) \text {; }\end{array}$ \\
\hline & & $\begin{array}{l}p< \\
0.0001\end{array}$ & $\begin{array}{l}p< \\
0.0001\end{array}$ & $\begin{array}{l}p< \\
0.0001\end{array}$ & 0.0001 & $\begin{array}{l}p< \\
0.0001\end{array}$ & $\begin{array}{l}p< \\
0.0001\end{array}$ & $\begin{array}{l}p< \\
0.0001\end{array}$ \\
\hline \multirow{3}{*}{\multicolumn{2}{|c|}{$\begin{array}{l}\text { Intraoperative } \\
\text { hypotension (MAP < } \\
60 \mathrm{mmHg})(1 / 0)\end{array}$}} & 2.66 & 2.58 & 2.54 & 2.52 & 2.42 & 2.38 & 2.65 \\
\hline & & $\begin{array}{l}(1.24- \\
5.67)\end{array}$ & $\begin{array}{l}(1.21- \\
5.49)\end{array}$ & $\begin{array}{l}(1.19- \\
5.41)\end{array}$ & $\begin{array}{l}(1.19- \\
5.37)\end{array}$ & $\begin{array}{l}(1.13- \\
5.18)\end{array}$ & $\begin{array}{l}(1.11- \\
5.12)\end{array}$ & $5.72)$ \\
\hline & & $\begin{array}{l}p= \\
0.0168\end{array}$ & $\begin{array}{l}p= \\
0.0139\end{array}$ & $\begin{array}{l}p= \\
0.0156\end{array}$ & $\begin{array}{l}P= \\
0.0163\end{array}$ & $\begin{array}{l}P= \\
0.0228\end{array}$ & $\begin{array}{l}P= \\
0.0262\end{array}$ & 0.0129 \\
\hline \multirow{3}{*}{\multicolumn{2}{|c|}{ AUROC }} & 0.837 & 0.839 & 0.836 & 0.835 & 0.840 & 0.842 & 0.852 \\
\hline & & $\begin{array}{l}(0.80- \\
0.87)\end{array}$ & $\begin{array}{l}(0.80- \\
0.87)\end{array}$ & $\begin{array}{l}(0.80- \\
0.87)\end{array}$ & $\begin{array}{l}(0.80- \\
0.87)\end{array}$ & $\begin{array}{l}(0.81- \\
0.87)\end{array}$ & $\begin{array}{l}(0.81- \\
0.87)\end{array}$ & $\begin{array}{l}(0.82- \\
0.88) ;\end{array}$ \\
\hline & & $\begin{array}{l}P< \\
0.0001\end{array}$ & $\begin{array}{l}P< \\
0.0001\end{array}$ & $\begin{array}{l}P< \\
0.0001\end{array}$ & $\begin{array}{l}P< \\
0.0001\end{array}$ & $\begin{array}{l}P< \\
0.0001\end{array}$ & $\begin{array}{l}P< \\
0.0001\end{array}$ & $\begin{array}{l}P< \\
0.0001\end{array}$ \\
\hline \multicolumn{9}{|c|}{ Values are presented as odds ratios (confidence intervals) and their ' $p$ ' values. } \\
\hline \multicolumn{9}{|c|}{$\begin{array}{l}\text { *Variables that failed to be significant in the multivariable models were as follows: } \mathrm{PP}>50 \mathrm{mmHg} \text {, } \\
\mathrm{PP}>55 \mathrm{mmHg}, \mathrm{PP}>60 \mathrm{mmHg} \text {, age, ASA III/IV/V, CCI, Adjunction of regional anaesthesia, procedure } \\
\text { risk (III), oncological procedure, catecholamine use, SBP }>160 \mathrm{mmHg}, \mathrm{DBP}<50 \mathrm{mmHg} \text {, SBP (per } 1 \\
\mathrm{mmHg} \text { ). }\end{array}$} \\
\hline
\end{tabular}

Figure 3. Pulse pressure thresholds and their relationship with hypoperfusion-related organ injury. The box represents odds ratio whereas the whiskers represent confidence intervals. "*” represents statistically significant values. 


\section{Discussion}

The main finding of our exploratory study is that increasing intraoperative values of pulse pressure were associated with the occurrence of hypoperfusion-related organ injury. This association persisted after adjusting for confounding factors (most importantly: high SBP and low DBP). We found a cut-off point of $>74 \mathrm{mmHg}$ of maximal PP to be associated with the outcomes. In regards to the dichotomous thresholds, PP above $65 \mathrm{mmHg}$ and onward was linked to hypoperfusion. Pulse pressure above 90 $\mathrm{mmHg}$, out of all PP thresholds applied, appeared to be the best predictor of hypoperfusion-related organ injury.

To our knowledge, this is the first study investigating the role of intraoperative pulse pressure in abdominal surgery in such a complex manner. It is known that increased ambulatory pulse pressure is strongly associated with cardiovascular events not only in general population but also in cardiac-surgery setting, irrespectively of the presence of chronic arterial hypertension $[6,22,23]$. Pulse pressure stands as a proxy for general vascular health and reflects cardiovascular risk better than isolated measurements of either systolic or diastolic pressure [24]. Generally, a value of PP is determined by stroke volume, left ventricle contractility and arterial compliance. Interestingly, pre-induction pulse pressure values (a reflection of baseline pulse pressure) alone were not significantly related to the outcome. In Abbot's and Mitrer's studies, it was found that increasing values of ambulatory and pre-induction PP values were significantly related to the increased occurrence of postoperative $\mathrm{MI}$ and $\mathrm{AKI}[5,25]$. It must be remembered, however, that those studies were performed among cardiac surgery patients with preexisting cardiac morbidities and the effect of preoperative pulse pressure might be more significant than in the non-cardiac setting. The fact that in our cohort pre-induction PP was not associated with hypoperfusion gave us more space to explore the impact of intraoperative values. Nevertheless, intraoperative PP positively correlated with pre-induction values. What's especially interesting, the negative correlation between pulse pressure and diastolic pressure was two times stronger in patients with the compromised outcome. Lowered DBP is known to decrease coronary perfusion and can could be associated with the development of AKI $[15,26,27]$.

We discovered that patients who experienced hypoperfusive outcome, exhibited higher values of PP and the odds ratios varied, depending on the threshold applied. Somewhat contrary to our hypothesis, Ahuja et al., in a large cohort of 23,000 patients, found that pulse pressure below $35 \mathrm{mmHg}$ was linked to postoperative $\mathrm{MI}$ and $\mathrm{AKI}$ [16]. Low pulse pressure is thought to predict cardiovascular events in patients with impaired cardiac function: decreased contractility of left ventricle causes SBP to achieve lower values and negatively impact the value of PP. It must be remembered that Ahuja et al. explored only the lowest values of PP and called for further research regarding high intraoperative pulse pressure.

High PP could influence systemic circulation in numerous ways. First, kidneys and brain have a high resting blood flow. With the increase of PP, perfusion of those organs becomes more pulsatile and it is thought to damage endothelium, smooth muscle and induce sheer stress which can cause plaque to rupture and form thrombosis [28-30] Additionally, high PP can decrease flow mediated vasodilation [31]. 
What is also worth mentioning is that increased PP causes aortic lumen to decrease which results in ventricular-aortic decoupling characterized by cardiac output that is too great to be accommodated by aortic lumen (leading to impaired cardiac output with preserved systolic function) [5, 32].

Above mentioned findings should be analysed with caution due to possible confounders. Firstly, the true association between high intraoperative pulse pressure and hypoperfusive outcome is, to certain extent, determined by the preoperative PP values. Despite pre-induction PP was not significantly related to the outcome in our analysis, it is possible that intraoperative PP is only a reflection of an overall cardiovascular condition and it does not impair organ perfusion in short-term period (such as duration of surgical procedure). Secondly, a pre-induction blood pressure value was defined as 'baseline' MAP. It is possible that such measurement does not represent the true 'baseline' as it could be influenced by stress or premedication. Thirdly, the blood pressure measurements were recorded in 5-minute intervals: a risk of underrecognition of pulse pressure changes exists. Additionally, we did not assess the level of preoperative organ injury that could be a result of e.g. prior chronic arterial hypertension. Moreover, we did not analyse any chronic hypotensive treatment. Finally, our analysis was restricted to a limited population of abdominal patients which reduces the generalizability of our results into all non-cardiac surgery settings.

\section{Conclusions}

High intraoperative pulse pressure is associated with hypoperfusion-related organ injury in patients undergoing abdominal surgery. However, the effect of high pulse pressure should be confirmed in other non-cardiac populations to prove generalizability of our results.

\section{Abbreviations}

AKI - Acute kidney injury

$\mathrm{Ml}$ - Myocardial infarction

PP - Pulse pressure

POQI - Perioperative Quality Initiative

MAP - mean arterial pressure

SBP - Systolic blood pressure

DBP - Diastolic blood pressure

$\mathrm{CCl}$ - Charlson Comorbidity Index

ASA - PS - American Society of Anesthesiology physical class 


\section{Declarations}

Ethics approval and consent to participate

1. The study was performed in accordance with the ethical standards of the Declaration of Helsinki (1964) and its subsequent amendments. All patients gave their written informed consent for data management. For this particular study, under Section 21 and 22 of the Act of 5 December 1996 on the Medical Profession in Poland, due to anonymous and non-interventional design of the project, no additional approval of the Ethics Committee was required (Act of Medical Profession in Poland 1996 https://isap.sejm.gov.pl/isap.nsf/download.xsp/WDU19970280152/O/D19970152.pdf) .The authors accessed data through administrative permissions given by the hospital director.

Consent for publication

Not applicable.

Availability of data and materials

Not applicable.

Competing interests

The authors declare that they have no competing interests.

Funding

None.

Authors' contributions

ZP and SC gathered all the necessary data. ZP, SC and $Ł J K$ analysed and interpreted data. ZP and SC prepared the manuscript. $Ł J K$ reviewed and corrected the manuscript.

Acknowledgements

Not applicable.

\section{References}

1. Salmasi V, Maheshwari K, Yang D, et al. Relationship between Intraoperative Hypotension, Defined by Either Reduction from Baseline or Absolute Thresholds, and Acute Kidney and Myocardial Injury after Noncardiac Surgery: A Retrospective Cohort Analysis. Anesthesiology. 2017;126:47-65.

2. Bijker JB, Persoon S, Peelen LM, et al. Intraoperative hypotension and perioperative ischemic stroke after general surgery, a nested case-control study. Anesthesiology. 2012;116:658-64. 
3. Wesselink EM, Kappen TH, Torn HM, et al. Intraoperative hypotension and the risk of postoperative adverse outcomes: a systematic review. Br J Anaesth. 2018;121:706-21.

4. McEvoy MD, Gupta R, Koepke EJ, et al. Perioperative Quality Initiative consensus statement on postoperative blood pressure, risk and outcomes for elective surgery. Br J Anaesth. 2019;122:57586.

5. Abbott TEF, Pearse RM, Archbold RA, et al. Association between preoperative pulse pressure and perioperative myocardial injury: an international observational cohort study of patients undergoing non- cardiac surgery. Br J Anaesth. 2017;119:78-86.

6. Cheng S, Xanthakis V, Sullivan LM, et al. Epidemiology / Population Science Blood Pressure Tracking Over the Adult Life Course Patterns and Correlates in the Framingham Heart Study 2012:1393-9.

7. Sessler DI, Bloomstone JA, Aronson S, et al. Perioperative Quality Initiative consensus statement on intraoperative blood pressure, risk and outcomes for elective surgery. Br J Anaesth. 2019;122:56374.

8. Czajka S, Putowski Z, Krzych ŁJ. Intraoperative hypotension and its organ-related consequences in hypertensive subjects undergoing abdominal surgery: a cohort study. Blood Press. 2021 Jul 29:1-11.

9. National Confidential Enquiry into Patient Outcome and Death. https://www.ncepod.org.uk/. Accessed 11 Jan 2021.

10. Doyle DJ, Goyal A, Bansal P, Garmon EH. American Society of Anesthesiologists Classification (ASA Class). 2020.

11. Kristensen SD, Knuuti J, Saraste A, et al. 2014 ESC/ESA Guidelines on non-cardiac surgery: cardiovascular assessment and management. Eur Heart J. 2014;35:2383-431.

12. Homan TD, Bordes S, Cichowski E. Physiology PP. [Updated 2021 Mar 31]. In: StatPearls [Internet]. Treasure Island (FL): StatPearls Publishing; 2021 Jan-. Available from: https://www.ncbi.nlm.nih.gov/books/NBK482408/.

13. Fontes ML, Aronson S, Mathew JP, et al. Pulse pressure and risk of adverse outcome in coronary bypass surgery. Anesth Analg. 2008;107:1122-9.

14. Blacher J, Evans A, Arveiler D, et al. Residual cardiovascular risk in treated hypertension and hyperlipidaemia: the PRIME Study. J Hum Hypertens. 2010;24:19-26.

15. Abbott TEF, Pearse RM, Archbold RA, et al. A Prospective International Multicentre Cohort Study of Intraoperative Heart Rate and Systolic Blood Pressure and Myocardial Injury After Noncardiac Surgery: Results of the VISION Study. Anesth Analg. 2018;126(6):1936-45.

16. Ahuja S, Mascha EJ, Yang D, et al. Associations of Intraoperative Radial Arterial Systolic, Diastolic, Mean, and Pulse Pressures with Myocardial and Acute Kidney Injury after Noncardiac Surgery: A Retrospective Cohort Analysis. Anesthesiology 2020;132:291-306.

17. Sun LY, Wijeysundera DN, Tait GA, et al. Association of intraoperative hypotension with acute kidney injury after elective noncardiac surgery. Anesthesiology. 2015;123:515-23. 
18. Kellum JA, Lameire N, Aspelin P, et al. Kidney disease: Improving global outcomes (KDIGO) acute kidney injury work group. KDIGO clinical practice guideline for acute kidney injury. Kidney Int. 2012;Suppl. 2:1-138.

19. Thygesen K, Alpert JS, Jaffe AS, ESC Scientific Document Group. et al. Fourth universal definition of myocardial infarction (2018). Eur Heart J. 2019;40(3):237-69.

20. Ringleb A, Bousser P, Ford M. G, et al. Guidelines for management of ischaemic stroke and transient ischaemic attack 2008 The European Stroke Organization (ESO) Committee and the ESO Writing Committee Executive. Stroke. 2008;25:6855-9.

21. von Elm E, Altman DG, Egger M, et al. STROBE Initiative. The Strengthening the Reporting of Observational Studies in Epidemiology (STROBE) Statement: guidelines for reporting observational studies. Int J Surg. 2014;12(12):1495-9.

22. Mancusi C, Losi MA, Izzo R, et al. Higher pulse pressure and risk for cardiovascular events in patients with essential hypertension: The Campania Salute Network. Eur J Prev Cardiol. 2018;25:235-43.

23. Howell SJ, Sear YM, Yeates D, et al. Hypertension, admission blood pressure and perioperative cardiovascular risk. Anaesthesia. 1996;51:1000-4.

24. Haider AW, Larson MG, Franklin SS, et al. Systolic blood pressure, diastolic blood pressure, and pulse pressure as predictors of risk for congestive heart failure in the Framingham Heart Study. Ann Intern Med. 2003;138:10-6.

25. Mitrev L, Speich KG, Ng S, et al. Elevated Pulse Pressure in Anesthetized Subjects Before Cardiopulmonary Bypass Is Associated Strongly With Postoperative Acute Kidney Injury Stage. J Cardiothorac Vasc Anesth. 2019;33:1620-6.

26. Assmann G, Cullen P, Evers T, et al. Importance of arterial pulse pressure as a predictor of coronary heart disease risk in PROCAM. Eur Heart J. 2005;26:2120-6.

27. Sato R, Luthe SK, Nasu M. Blood pressure and acute kidney injury. Crit Care. 2017;21:28.

28. O'Rourke MF, Safar ME. Relationship between aortic stiffening and microvascular disease in brain and kidney: cause and logic of therapy. Hypertens (Dallas, Tex 1979) 2005;46:200-4.

29. Lyon RT, Runyon-Hass A, Davis HR, et al. Protection from atherosclerotic lesion formation by reduction of artery wall motion. J Vasc Surg. 1987;5:59-67.

30. Traub O, Berk BC. Laminar shear stress: mechanisms by which endothelial cells transduce an atheroprotective force. Arterioscler Thromb Vasc Biol. 1998;18:677-85.

31. Ceravolo R, Maio R, Pujia A, et al. Pulse pressure and endothelial dysfunction in never-treated hypertensive patients. J Am Coll Cardiol. 2003;41:1753-8.

32. Mitchell GF, Lacourcière $Y$, Ouellet J-P, et al. Determinants of elevated pulse pressure in middle-aged and older subjects with uncomplicated systolic hypertension: the role of proximal aortic diameter and the aortic pressure-flow relationship. Circulation. 2003;108:1592-8.

\section{Figures}




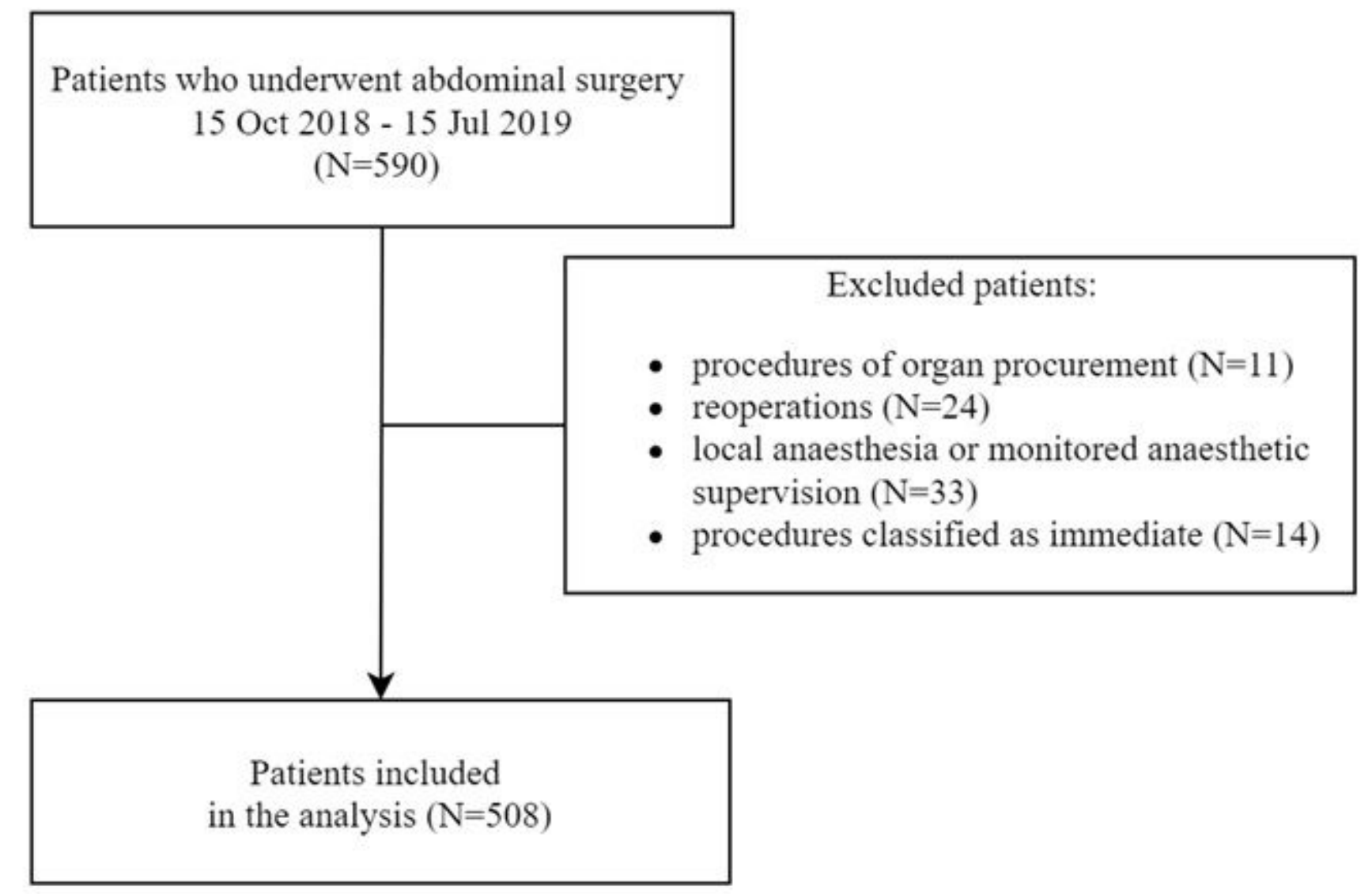

Figure 1

Flow diagram for the patient selection process 


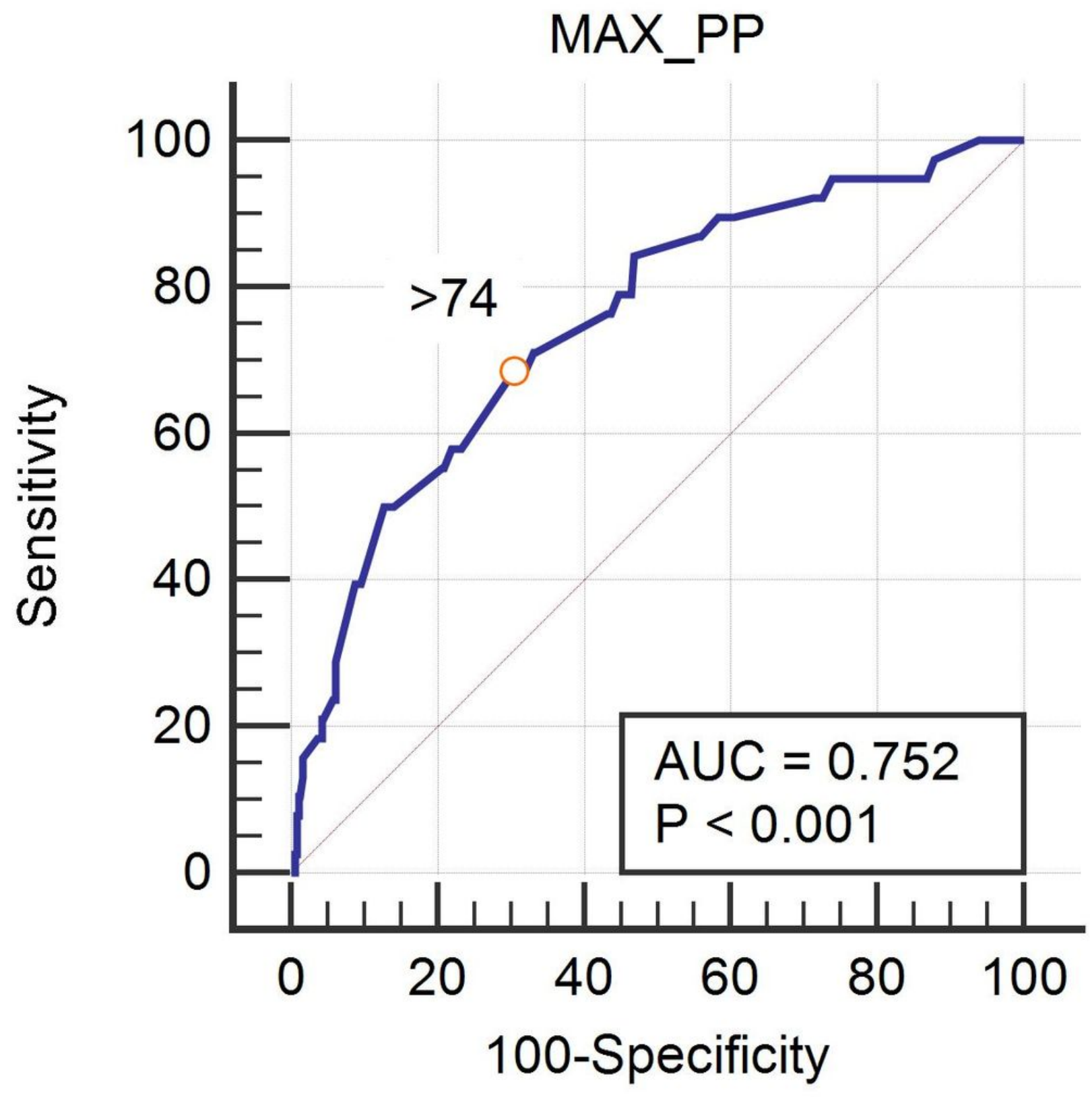

Figure 2

ROC curve analysis of maximal PP values registered over the course of procedure. 


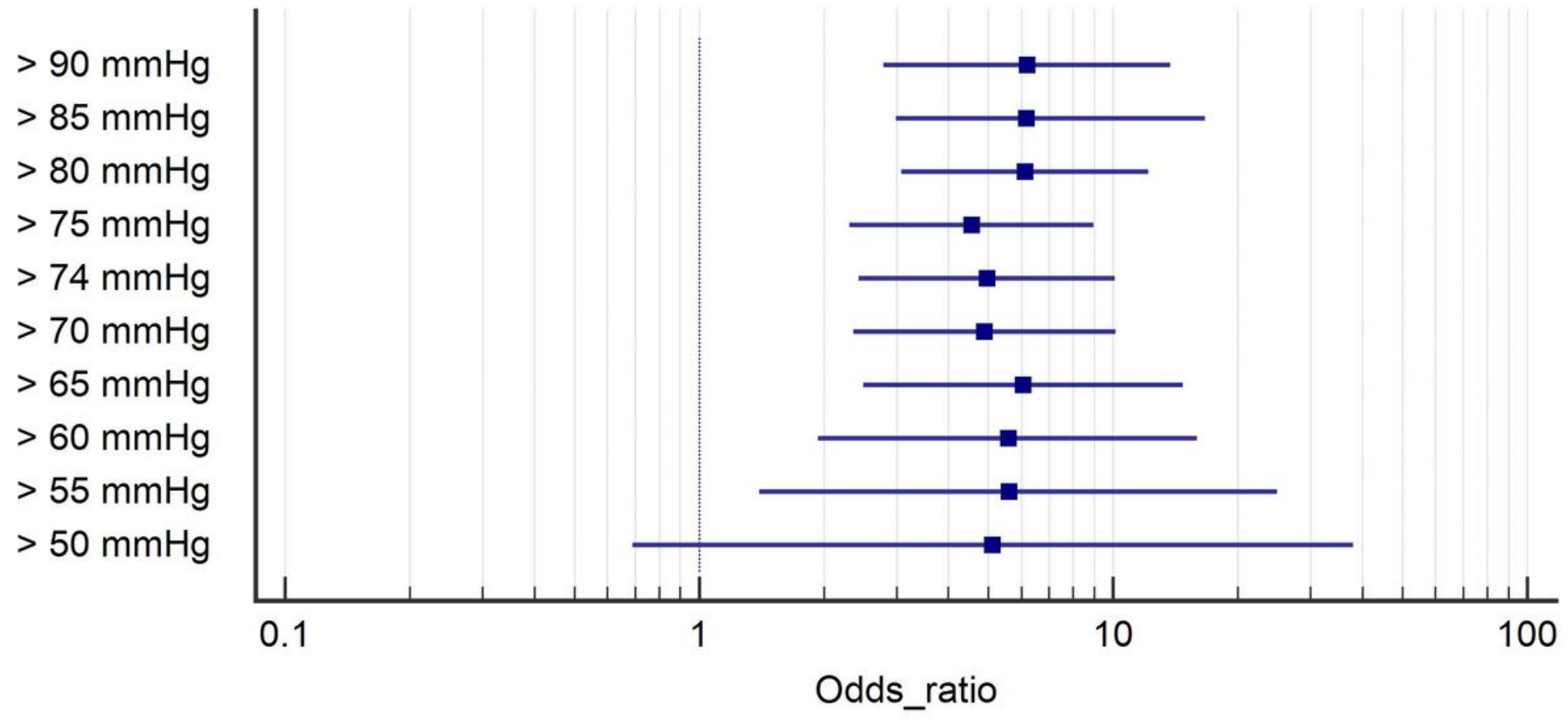

\section{Figure 3}

Pulse pressure thresholds and their relationship with hypoperfusion-related organ injury. The box represents odds ratio whereas the whiskers represent confidence intervals. " $\star$ " represents statistically significant values.

\section{Supplementary Files}

This is a list of supplementary files associated with this preprint. Click to download.

- STROBEchecklistcohort.doc 